\title{
Application of Particle Swarm Optimization Algorithm in Talent Policy System Optimization
}

\author{
Lei Yang ${ }^{1, a}$, Yang Yang $\mathbf{L i}^{2, b}$ \\ ${ }^{1}$ School of Automation, Northwestern Polytechnical University, Xi'an, China 710072 \\ ${ }^{2}$ School of Mingde, Northwestern Polytechnical University, Xi'an, China 710072 \\ 3332749268@qq.comb675744172@qq.com
}

Keywords: Talents Policy System, Chaotic Particle Swarm Optimization, Policy Factors

\begin{abstract}
As a macro-management system, the complexity of the talent policy system is reflected on that the evaluation results of policy factors are hard to quantify, and the mismatching between the system optimization direction and the social and psychological requirements of talents, et al. In order to solve above problems, a Shaanxi province talents policy system is used as example, a questionnaire about policy satisfaction, engagement and demission tendency is designed and the questionnaire data are collected by using empirical survey method. Based on the questionnaire data, the chaotic particle swarm optimization (CPSO) algorithm is used to build the relationship model for talent policy system, i.e. the mathematical model of the talent policy system. By analyzing the gain coefficient of the model, the contribution rate of different talents policy for the policy satisfaction, engagement and the demission tendency can be obtained. The simulation results show that, compared with the traditional regression approach to build the mathematical model of the talent policy system, the CPSO method has high accuracy, low complexity for computer realization and can be extended to the optimization of other policy systems.
\end{abstract}

\section{Introduction}

The flow rules of the talents inside or outside the country show that, good institutional mechanisms have more conducive to the growth and function exertive of the talents [1]. Therefore, how to adapt the trends of industrial structure optimization and upgrade, and continual enhance the attraction of talents, are the reality and urgent project. The modeling and controlling of the talents policy system became very important research issues.

\section{Building of the Relationship Model for the Talent Policy System}

In this paper, a Shaanxi province talents policy system is used as example, a questionnaire is designed,that the effects of different policy factors to the talent satisfaction, engagement and demission tendency are collected. From the questionnaire data, the relationship model between the different policy factors and the talents policy satisfaction can be expressed as 


$$
S=X_{1} * P_{1}+X_{2} * P_{2}+X_{3} * P_{3}+X_{4} * P_{4}+X_{5} * P_{5}+X_{6} * P_{6}+X_{7} * P_{7}+X_{8} * P_{8}+X_{9} * P_{9}+X_{10} * P_{10}
$$

where $S$ denotes the mean value of talents policy satisfaction, $X_{\mathrm{i}}(\mathrm{i}=1, \ldots, 10)$ represent the satisfaction gain coefficient of the different policy factors, $X_{i}$ is bigger means that the corresponding policy factor has larger contribution for the talent policy satisfaction, otherwise the contribution is smaller. $P_{1}$ to $P_{10}$ represent satisfaction of different policy factors, shown in Table1:

Table $1 P_{1}$ to $P_{10}$ represent satisfaction of different policy factors

\begin{tabular}{|c|c|c|c|}
\hline Variable & Policy Factor & Variable & Policy Factor \\
\hline$P_{1}$ & management & $P_{6}$ & culture policy \\
\hline$P_{2}$ & living environment & $P_{7}$ & social atmosphere and respected \\
\hline$P_{3}$ & talent evaluation & $P_{8}$ & introduction policy \\
\hline$P_{4}$ & incentives policy & $P_{9}$ & talent market service \\
\hline$P_{5}$ & regional flow policy & $P_{10}$ & policy awareness \\
\hline
\end{tabular}

It is obvious, the key problem of the talents policy satisfaction relationship model buliding process, is calculate the gain coefficient $X_{\mathrm{i}}$ according the questionnaire data.

The traditional regression approach, but the regression model data needs to meet the effect accumulation, without variable measurement error and other multiple assumptions, and that the test process is complicated [2], so we use a nevol CPSO method [3,4] to product the multi-objective gain $X_{i}$ in Eq.(1), thus the relationship model of the talents policy satisfaction system is built and the improved policy recommendations are proposed, this is the main motivation of the paper.

\subsection{CPSO method for Talents Policy System Optimization}

In the traditional Particle Swarm Optimization (PSO) method[5], the distribution of the initial particle has significant effect on performance of the optimizing algorithm, the uneven distribution initial particles may lead the algorithm instability, CPSO method, can initialize particles to even distribution by using the chaos map function. In this paper, an improved CPSO method[3] is used for talents policy system optimization, the improved CPSO method, itproposed to initialize the initial particles by using the unilateral coupled lattice spatiotemporal chaotic map, the chaotic map is expressed as

$$
\left.L_{n}(m+1)=\left(1-\varepsilon_{n}\right) f\left[L_{n}(m)\right]+\varepsilon_{n} f\left[L_{\mathrm{n}-1}(m)\right] n=1,2,3, . .\right]
$$

where $\mathrm{f}\left[L_{n}(m)\right.$ is chaotic $\operatorname{map}_{2} f\left[L_{n}(m)\right]=\mu L_{n}(m)\left(1-L_{n}(m)\right), L_{n}(m)$ denotes the particle, $n$ is the dimension of particle (space), $m$ represents the population length of particle (discrete-time), $\varepsilon_{n}$ is the coupling strength. In Eq. (2), the initial particles are directly produce, which not only satisfy the chaos distributionin space (dimension), but also satisfy the chaos distribution in discrete-time, the unilateral coupled lattice spatiotemporal chaotic map is more sensitive to the initial value, and with more stringent boundary conditions, thus has better performance.The unilateral coupled lattice spatiotemporal chaotic map diagram shown in Fig.1: 


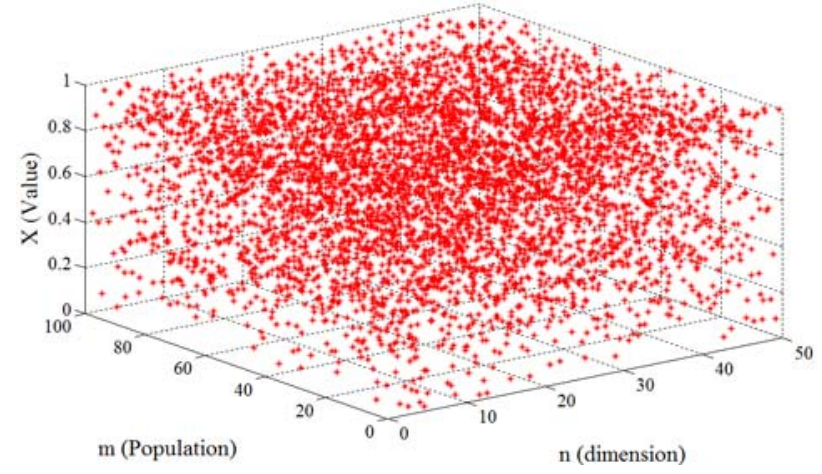

Fig.1. The state diagram of unilateral coupled lattice spatiotemporal chaotic time space

The traditional CPSO methods use logistic map, Figure 3 shows the distribution comparison results between the traditional logistics map and the unilateral coupled lattice spatiotemporal chaotic map. Figure 3(a) and (b) are the box-plot curve of the proposed method and the logistic map respectively. Figure 3(c) is the comparison results of $N$-dimension elements between the proposed chaotic map and the traditional logistic map when $m=30$. From Fig.3, we know that, the multi-dimensional particles, generated by the proposed chaotic map, are uniformly distributed in the searching space, and compared with the traditional logistic map, the proposed method are more evenly distributed in $N$-dimension space from the box-plot curve. From Fig.3(c), we can see that, compared to the traditional logistic map, the proposed method has tighter boundary conditions in the sense of no boundary point as appear in "red star points" in the left subplot of Fig.2(c) (by using the logistic map)

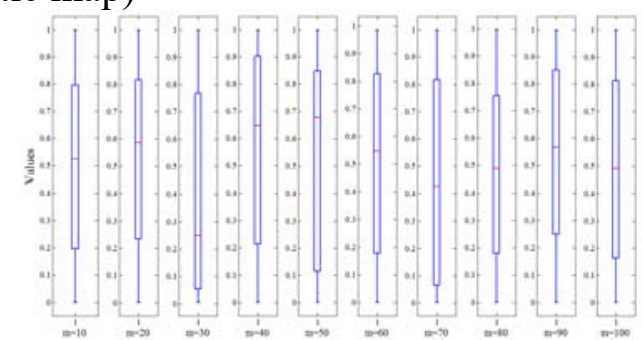

(a)

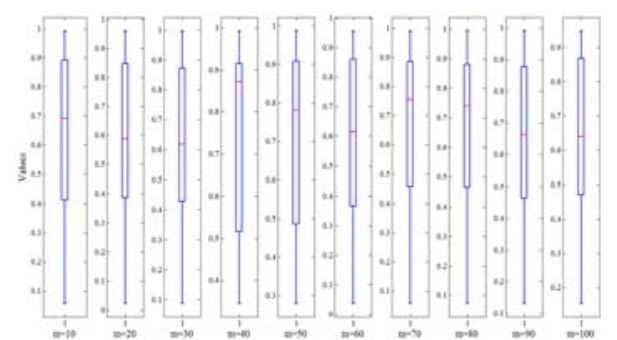

(b)
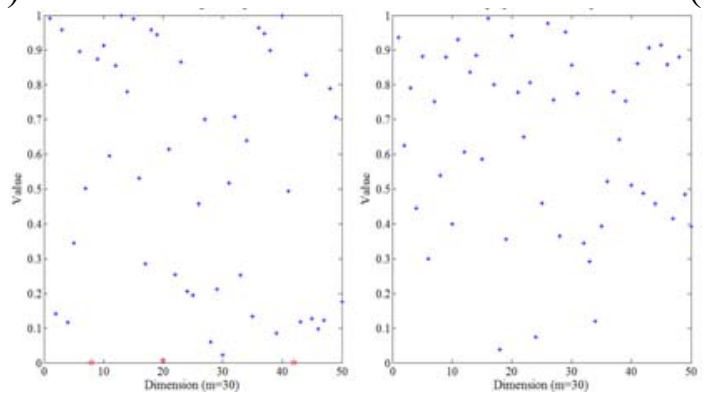

(c)

Fig.2. Comparison of different chaotic map (a) the proposed chaotic map;(b) logistic maps;(c) $N$-dimensional element distribution comparison when $m=30$.

Using the novel CPSO method, the relationship model of the talents policy satisfaction system can be obtained. The gain coefficient $X_{i}$ are treat as the particles, from Eq. (1), there are 10 gain coefficients, thus the dimension $N$ of particle is 10 . In the $k$-th iteration, the particle value $X_{m}$ is obtained, and the cost function, which presents the error between the actual mean talent satisfaction value collect by questionnaire and the estimated value calculate by Eq. (1), is calculated. Using the following equation, the velocity and position of $n$-dimensional $(n=1, \ldots, N)$ particles $m$ can be updated. 


$$
\begin{aligned}
& V_{m}(n, k+1)=V_{m}(n, k)+c 1 * r 1 *\left(Y_{m}(n, k)-X_{m}(n, k)\right)+c 2 * r 2 *\left(Y_{g}(n, k)-X_{m}(n, k)\right) \\
& X_{m}(n, k+1)=X_{m}(n, k)+V_{m}(n, k+1)
\end{aligned}
$$

where $V_{m}$ denotes the speed of particle, $Y_{m}$ denotes the individual extreme value, $Y_{g}$ denotes the global extreme value, $c 1, c 2$ is learning factor, non-negative constant, $r 1, r 2$ is the two independent random numbers between $[0,1]$.The detailed optimization process is showing blow:

Step1, determine the parameters to be optimized is the coefficient $X_{i}(i=1, \ldots, 10)$ in formula (1), the values of $X_{i}$ are the $N$-dimensional particle positions, $N=10$, initialized the population size of particles is $M=10000$. The maximum number of iterations is $k_{\max }=50$. Using Eq. (2) unilateral coupled lattice spatiotemporal chaotic map to initialize $N$-dimensional particle position $X_{m}(:, 0)=$ $\left[X_{m}(1,0), \ldots, X_{m}(10,0)\right] \in[0,1], m=1, \ldots, M$; Similarly initialize the global particle best position $Y_{g}(0)=\left[Y_{g}(1,0), \ldots, Y_{g}(10,0)\right] \in[0,1]$.

Step2, start the process of iteration by running algorithm optimization program, the coefficients $\left(X_{m}(1, k)=X_{1}, \ldots, X_{m}(10, k)=X_{10}\right)$ represents the current $k$ iteration particle $m$ position, take $X_{m}$ $(1, k)$ into Eq. (1), where $k=1, \ldots, k_{\max }$. The calculation results of particle $m$ corresponding to different coefficients in $k$ iteration expressed as:

$$
S_{\mathrm{g}}(m)=X_{m}(1, k)^{*} P_{1}+X_{m}(2, k)^{*} P_{2}+X_{m}(3, k)^{*} P_{3}+X_{m}(4, k)^{*} P_{4}+X_{m}(5, k)^{*} P_{5}+X_{m}(6, k)^{*} P_{6}+X_{m}(7,
$$
$k)^{*} P_{7}+X_{m}(8, k) * P_{8}+X_{m}(9, k)^{*} P_{9}+X_{m}(10, k)^{*} P_{10}$.

Thus, the objective function $J(m, k)=1 /\left(S-S_{g}(m)\right)$ can be calculated, where $S$ is the mean value of the talents policy satisfaction collect from actual questionnaire, $P_{1}$ to $P_{10}$ is talent policy factors average value also collect from actual questionnaire.

Step3, for each particle $m$ in $k$ iteration, $(m=1, \ldots, M)$, compare the objective function value of current particle with the objective function value of individual extreme particle, if the present objective function value is better than the objective function value of individual extreme particle, the current particle become the new individual extreme particle. This process is expressed as:

If $J(m) \geq J_{\max }(m)$, then $J_{\max }(m)=J(m, k)$, and $Y_{m}(:, k)=\left[Y_{m}(1, k), Y_{m}(2, k), \ldots, Y_{m}(N, k)\right]$.

Step4, for the $k$ iteration particle $m(m=1, \ldots, M)$, update particles speed and position according to individual extreme value $Y_{m}$ and global extreme value $Y_{g}$ of all the particles. The first iteration $(k=1)$, the global extreme value $Y_{g}(0)$ using initializing random value in Step1, in each later iteration, the global extreme value is calculated from step 5 in the last iteration.

Step5, comparing all the objective function value of $M$ particles, (saved the particle which the objective function is maximum of $M$ particles as the global extreme value.This process is expressed as: $J_{g}(m, k)=\max \{J(1, k), \ldots, J(M, k)\}$; then particle $M$ is the global optimal solution, expressed as $Y_{g}(:, k)=\left[Y_{g}(1, k), Y_{g}(2, k), Y_{g}(N, k)\right]=[X(1, k), X(2, k), \ldots, X(N, k)]$.

Step6, if reaches the stop condition $\left(k=k_{\max }\right)$, end the program and display the global extreme value $Y_{g}$ and the corresponding objective function value, otherwise $k=k+1$ and back to step 1 to continue the iterative process.

\subsection{Optimization Results Analysis}

From above steps, which is programed by Matlab,the relationship model of policy factors satisfaction and overall satisfation is given as:

$S=0.030665 * P_{1}+0.082074 * P_{2}+0.26538 * P_{3}+0.12782 * P_{4}+0.002439 * P_{5}+0.14332 * P_{6}+0.16516 *$

$P_{7}+0.13447 * P_{8}+0.035168 * P_{9}+0.1017 * P_{10}$

Similarly, we can build other relationship models such as the relationship model of policy factors 
satisfaction and demission tendency. Compared with the results, establish traditional regression equation need to extract the coefficient of correlation>0.6. By using SPSS17.0, we got the larger coefficient computing result shown as Table2:

Table 2 the larger coefficient computing result

\begin{tabular}{|cc|c|c|c|c|c|c|c|c|c|c|c|}
\hline & & $P_{1}$ & $P_{2}$ & $P_{3}$ & $P_{4}$ & $P_{5}$ & $P_{6}$ & $P_{7}$ & $P_{8}$ & $P_{9}$ & $P_{10}$ & Overall \\
\hline$P_{1}$ & Pearson & 1 & $.700^{*}$ & $.716^{*}$ & $.273^{*}$ & $.632^{*}$ & $.943^{*}$ & -.25 & -.018 & -.114 & -.192 & $.594^{* *}$ \\
& Sig. & & .000 & .000 & .003 & .000 & .000 & .004 & .430 & .12 & .27 & .000 \\
& $\mathrm{~N}$ & 102 & 102 & 102 & 102 & 102 & 102 & 102 & 102 & 102 & 102 & 102 \\
\hline$P_{3}$ & Pearson & $.716^{*}$ & $.375^{*}$ & 1 & $.275^{*}$ & $.444^{*}$ & $.622^{*}$ & -.132 & $.284^{*}$ & -.081 & -.293 & $.555^{* *}$ \\
& Sig & .000 & .000 & & .003 & .000 & .000 & .092 & .002 & .21 & .00 & .000 \\
& $\mathrm{~N}$ & 102 & 102 & 102 & 102 & 102 & 102 & 102 & 102 & 102 & 102 & 102 \\
\hline
\end{tabular}

The largest coefficient is 0.594 , it does not meet the requirements of the regression model, to avoid error accumulation, the regression model cannot be established.

\section{Summary}

By using advanced particle swarm optimization algorithm, the establishment of complex talent policy system model can be achieved, which provides basis for analyzing the role of policy and proposing the optimization proposal. The particle swarm optimization algorithm has high accuracy, low complexity for computer realization and can be extended to the optimization of other management systems.

\section{Acknowledgements}

The present work is supported by the fund of Shaanxi Provincial Natural Science (2014JM2713)

\section{References}

[1] Le yuan Guan: Talent Strategy Explore in the New Century (Central Party School Publications, China 2006) [2]Cheng ou Wu, Liang wei Qin: Modern practical Multivariate statistical analysis (Meteorological Publications, China 2007)

[3]Xin Guo, Haipeng Ren*, Ding Liu: Optimized PI Controller Design for Three Phase PFC Converter Based on Multi-Objective Chaotic Particle Swarm Optimization. Journal of Power Electronics, 2016, 16(2): P.610-620

[4]C. A. Coello,D. A. V. Veldhuizen, G. B. Lamont: Evolutionary algorithms for solving multi-objective problems(Kluwer Academic Publishers, Netherlands 2002)

[5]Hai-Peng Ren*, XinGuo:Optimization Controller Design of CACZVS Three Phase PFC Converter Using Parti cle Swarm Optimization.In proceedings of 40th Annual Conference on IEEE Industrial Electronics Society, IECO N 2014, Dallas, USA 